\title{
Desempeño del tutor de medicina en una universidad pública Medicine Tutor Performance at a Public University Tutor desempenho a um medicamento universidade pública
}

\author{
Josefina Salomón Cruz \\ Universidad Juárez Autónoma de Tabasco, México \\ josefinasac@hotmail.com \\ María Luisa Castillo Orueta \\ Universidad Juárez Autónoma de Tabasco, México \\ malucaota@hotmail.com \\ Juan Antonio Córdova Hernández \\ Universidad Juárez Autónoma de Tabasco, México \\ uan.cordova@live.com.mx
}

\section{Resumen}

La tutoría es una estrategia que busca alcanzar la formación integral de los estudiantes, la calidad en la educación y la disminución de indicadores tales como deserción, rezago y reprobación escolar. Por ello, la función del tutor representa un compromiso docente e institucional. El objetivo de este estudio es evaluar el desempeño de los tutores en la licenciatura de médico cirujano de la división académica de ciencias de la salud de la Universidad Juárez Autónoma de Tabasco a través de la percepción de los tutorados. El material y los métodos utilizados consistieron en un estudio cuantitativo, descriptivo, transversal y prospectivo, con un universo de 2008 tutorados y una muestra de 335 inscritos en el periodo febrero-agosto 2015; para la encuesta se utilizó un cuestionario con Alfa de Cronbach de 0.97. En los resultados se valoraron dos aspectos del desempeño del tutor: el académico y el afectivo; el primero fue bueno y el segundo regular de acuerdo a la percepción de los tutorados. 
Palabras clave: tutoría, desempeño, tutor.

\section{Abstract}

Tutoring is a strategy that seeks to achieve the comprehensive training of students, the quality of education and the decrease in indicators such as desertion, lagging and failure. Therefore, the function of Tutor represents a teaching and institutional commitment. The objective of this study is to evaluate the performance of the tutors in the degree of Surgeon of the Academic Division of Health Sciences of Juárez Autonomous University of Tabasco through the perception of the tutees. the material and methods used consisted of a quantitative, descriptive, transversal and prospective study, with a universe of 2008 tutees and a sample of 335 registered in the period February-August 2015; for the survey was used a questionnaire with Cronbach's alpha of 0.97. For Results were evaluated two aspects of the performance of the Tutor: the academic and the affective; the first was good and the second regular according to the perception of the tutees.

Key words: tutoring, performance, tutor.

\section{Resumo}

Mentoring é uma estratégia que tem como objectivo a formação integral dos alunos, educação de qualidade e declínio indicadores, como evasão, atraso e fracasso escolar. Portanto, o papel do professor é um professor e compromisso institucional. O objetivo deste estudo é avaliar o desempenho dos tutores na divisão cirurgião acadêmica de graduação de ciências da saúde da Universidade Juárez Autônoma de Tabasco através da percepção de médico tutelado. O material e os métodos utilizados consistiram de um quantitativo, descritivo, transversal e um estudo prospectivo, com um universo de 2.008 mentees e uma amostra de 335 registrado no período fevereiro-agosto de 2015; para o questionário da pesquisa com alfa de 0,97 de Cronbach foi utilizado. Nos resultados foram avaliados dois aspectos do desempenho do tutor: acadêmica e afetiva; o primeiro foi boa ea segunda regulada de acordo com a percepção dos orientandos.

Palavras-chave: tutoria, desempenho, tutor. 


\section{Introducción}

La tutoría es una actividad que actualmente se considera indispensable para la formación integral de los estudiantes. La actividad tutorial en México tiene su origen en posgrado, en la Facultad de Química de la Universidad Nacional Autónoma de México (UNAM). A nivel licenciatura, el sistema tutorial inició también en la UNAM en el Sistema de Universidad Abierta. Poco a poco, a partir del año 2000, el Sistema Tutorial se fue implementando en las Instituciones de Educación Superior (IES), a partir de las recomendaciones emitidas por la Asociación Nacional de Universidades e Instituciones de Educación Superior (ANUIES, 2000).

Existen variados conceptos de tutoría. En México, la ANUIES la define como un acompañamiento y atención personalizada del docente hacia el alumno que favorece su adaptación al ambiente universitario, su formación y el logro de los objetivos académicos; además, la propuso como una estrategia fundamental para alcanzar la calidad de la educación superior, disminuir la deserción, el rezago, la reprobación escolar y aumentar la eficiencia terminal (ANUIES, 2000).

La Universidad Juárez Autónoma de Tabasco (UJAT) retomó la propuesta implementando el Programa Institucional de Tutorías (PIT), cuya misión es contribuir en la formación integral del alumno, a través de tutores que brinden orientación personalizada para el desarrollo profesional y personal con equilibrio en los aspectos cognitivos y socio-afectivos (Universidad Juárez Autónoma de Tabasco, 2003).

Este programa se aplicó en la licenciatura de médico cirujano de la división académica de ciencias de la salud (DACS) de la UJAT en el año 2006, y desde entonces no se había efectuado un estudio de evaluación del mismo, por lo que el objetivo de esta investigación fue evaluar el desempeño de los tutores de la licenciatura en médico cirujano de la DACSUJAT, de acuerdo a la percepción de los estudiantes, con la finalidad de implementar acciones de mejora que impacten en los indicadores de calidad del Programa Institucional 
de Tutorías y de Medicina (División Académica de Ciencias de la Salud, 2012). En la actualidad, el Programa de Tutoría (PT) de médico cirujano cuenta con 47 profesores, quienes fungen como tutores atendiendo una matrícula de 2008 estudiantes. Cada profesor tutor atiende en promedio a 40 tutorados realizando la tutoría en la modalidad individual durante la primera fase de la trayectoria académica del tutorado hasta alcanzar $45 \%$ del total de los créditos o en la modalidad grupal cuando el tutorado cursó más del $45 \%$ de créditos del total que conforman el programa educativo.

Ser tutor significa ser maestro, guía, defensor, protector, alguien que debe ser reconocido por su actitud y conocimiento, además de otras capacidades psicopedagógicas y científicas (Caballero, y Añorga, 2007), siendo algunas evaluadas por este estudio.

De acuerdo con varios autores, las tutorías tienen grandes beneficios. Lobato e Ilvento (2013), señalaron que favorecen la resolución de problemas personales y profesionales de los estudiantes. Para Waldeck, Orego, Plax y Kearney favorecen el éxito académico de los tutorados; para Campbell y Campbell benefician el aspecto académico, ayudan a la resolución de problemas y a satisfacer a los estudiantes (De la Cruz, Chehaybar y Abreu, 2011).

Por tanto, la acción tutorial tiene fuertes implicaciones en el desarrollo integral del estudiante, de ahí la importancia de identificar su percepción acerca del desempeño del tutor en cuanto a su actitud y conocimiento, como una dimensión para evaluar el Programa Institucional de Tutorías.

Por otro lado, el término desempeño, según la Real Academia Española (2016), significa el acto de desempeñarse, y este a su vez de ejercer las obligaciones inherentes a una profesión, cargo u oficio. Es un concepto empresarial definido por múltiples autores, entre ellos Chiavenato (2000), quienes lo conciben como las acciones y comportamientos observados en los empleados. En el aspecto académico, se habla de atributos para ejercer la tutoría y de buenos o malos tutores. Entre los primeros destacan los formativos (conocimiento de la disciplina), los didácticos (conocimiento en docencia), los interpersonales (disponibilidad, habilidades de comunicación y socialización) y los éticos (honestos y responsables) (De la Cruz, Chehaybar y Abreu, 2011).

En la actividad tutorial, la figura del tutor es de suma importancia debido a que de su buen desempeño dependen muchos beneficios que recibirán los estudiantes que participan en el 
programa. El tutor es la persona que puede apoyar a los alumnos a solucionar sus problemas de aprendizaje, desarrollar actitudes positivas, mejorar su aprendizaje y tomar buenas decisiones a corto y largo plazo, al concientizarlos y proporcionarles orientación sobre el panorama de su futuro profesional (Narro, 2013).

En esta investigación se valoraron los aspectos académico y afectivo en la formación del tutor y su función de acuerdo a los tutorados.

\section{METODOLOGÍA}

Se realizó un estudio de enfoque cuantitativo, descriptivo, transversal y prospectivo durante el periodo escolar febrero-agosto del 2015 en la licenciatura de médico cirujano de la DACS-UJAT, cuyo objeto de estudio fueron los tutorados que estaban cursando dicha licenciatura. El universo fue de 2008 alumnos, con una muestra probabilística de nivel de confianza de $95 \%$ y un error estándar de $5 \%$ en 335 estudiantes inscritos en el periodo que se recabaron los datos. El muestreo fue aleatorio simple para los semestres $2^{\circ}, 4^{\circ}, 6^{\circ}, 8^{\circ}$ y $10^{\circ}$.

La recolección de datos se realizó mediante la técnica de encuesta, con un instrumento que agrupó ítems del cuestionario de evaluación del Programa Institucional de Tutorías de la UJAT (2003), de la Universidad Nacional Autónoma de México (2012) y del instrumento utilizado para el mismo fin por los autores Marín y Sansores (2014) en la Universidad Autónoma de Quintana Roo. Este constó de dieciséis preguntas cerradas en escala tipo Likert ( $4=$ totalmente de acuerdo, $3=$ de acuerdo, $2=$ en desacuerdo y $1=$ totalmente en desacuerdo), con las cuales se indagó sobre los aspecto académico y afectivo del desempeño de los tutores.

El cuestionario se validó en prueba piloto con 60 estudiantes y un Alfa de Cronbach de 0.97 .

Los indicadores que se valoraron se agruparon en dos categorías: (1) la académica, que incluyó la formación del tutor basada en el conocimiento y dominio del acto de tutelar, mediante preguntas relacionadas con la capacidad de planeación, evaluación, realimentación y seguimiento de trayectorias; (2) la afectiva, que valoró la actitud del tutor a través del compromiso, disposición, asistencia, localización, interés, respeto, atención y comunicación. Los criterios de desempeño se valoraron con la siguiente escala: 1-25 \% mal 
desempeño, 26-50 \% desempeño regular, 51-75 \% buen desempeño y 76 -100 \% muy buen desempeño.

Para la valoración de la actitud de los tutores se consideró una escala similar: 1-25 \% muy mala actitud, 26-50\% regular actitud, 51-75\% buena actitud y 76-100\% muy buena actitud.

Para el procesamiento de la información se utilizó el paquete estadístico Statistical Packgage for Social Sciences (SPSS) versión 21 y se analizaron los datos con estadísticas descriptivas.

\section{RESULTADOS}

Se aplicó el cuestionario a 335 estudiantes, cuya edad promedio fue de 21 años, la muestra por sexo indicó que $53 \%$ (179) de los entrevistados fueron mujeres y 47 \% (156) hombres. Con respecto al número de créditos cursados, estuvieron más representados los estudiantes que habían cursado 0-20\%, seguidos del 61-80 \% de los créditos (gráfica 1).

\section{Gráfica 1: Distribución de tutorados según los créditos cursados}

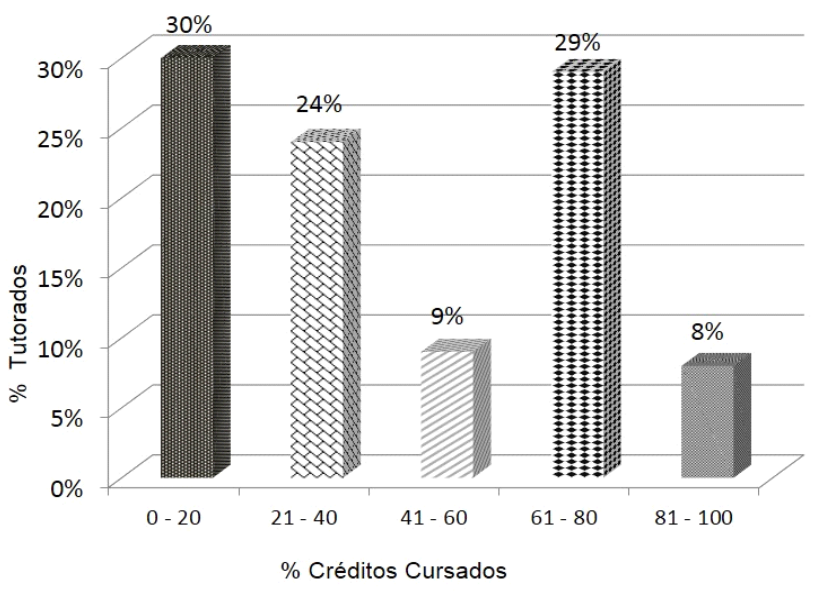

Fuente: datos recopilados por los autores (2015). 


\section{CATEGORÍA ACADÉMICA}

Esta categoría estuvo representada por nueve preguntas que abarcaron: el conocimiento de la normatividad, la orientación para resolver problemas de aprendizaje de los tutorados y las actividades realizadas por el tutor (tablas I y II).

En las preguntas orientadas a la formación del tutor se obtuvieron resultados ubicados en la escala del $51 \%$ al $75 \%$, es decir, un buen desempeño (tabla I).

Es importante remarcar que más del $30 \%$ de los tutorados perciben un desempeño regular del tutor en relación al conocimiento y la orientación.

Tabla I: Indicador de formación del tutor.

\begin{tabular}{lccc}
\hline & $\begin{array}{c}\text { Totalmente de } \\
\text { acuerdo } \\
\mathbf{f}(\%)\end{array}$ & $\begin{array}{c}\text { De } \\
\text { acuerdo } \\
\mathbf{f}(\%)\end{array}$ & $\begin{array}{c}\text { Total } \\
\mathbf{f}(\%)\end{array}$ \\
\hline $\begin{array}{l}\text { Al plantearle dudas académicas a tu tutor } \\
\text { percibiste que conoce la normatividad de la } \\
\text { institución. }\end{array}$ & $116(35)$ & $110(33)$ & $226(68)$ \\
\hline $\begin{array}{l}\text { Consideras que la formación de tu tutor } \\
\text { permitió una orientación adecuada para } \\
\text { resolver tus dificultades de aprendizaje. }\end{array}$ & $99(30)$ & $110(33)$ & $209(63)$ \\
\hline
\end{tabular}

Fuente: datos recopilados por los autores (2015).

La formación del tutor también fue valorada de acuerdo a las actividades que realiza con los tutorados, en este aspecto los resultados mostraron un desempeño bueno ya que de las siete preguntas realizadas todas estuvieron en una escala mayor a $51 \%$, siendo el porcentaje más alto $64 \%$ (que suma totalmente de acuerdo y de acuerdo), es decir, la actividad donde el tutor identifica los problemas y necesidades del tutorado que lo guían en su trayectoria académica (tabla II). 
Tabla II: Evaluación de las actividades de los tutores de medicina según los estudiantes

\begin{tabular}{lccc}
\hline \multicolumn{1}{c}{ Actividades de los tutores } & $\begin{array}{c}\text { Totalmente } \\
\text { de acuerdo } \\
\text { f \% }\end{array}$ & $\begin{array}{c}\text { De } \\
\text { acuerdo } \\
\text { f\% }\end{array}$ & f \% \\
\hline $\begin{array}{l}\text { Tu tutor identificó tus problemas y necesidades para } \\
\text { guiarte en tu trayectoria académica. }\end{array}$ & $92(28)$ & $122(36)$ & $214(64)$ \\
\hline $\begin{array}{l}\text { Consideras que la atención personalizada con tu tutor } \\
\text { te ha servido para establecer metas claras y factibles. }\end{array}$ & $95(28)$. & $104(31)$ & $199(59)$ \\
\hline $\begin{array}{l}\text { Tu tutor te da información oportuna y actualizada para } \\
\text { visualizar tus posibilidades profesionales. }\end{array}$ & $81(24)$ & $99(30)$ & $180(54)$ \\
\hline $\begin{array}{l}\text { Tu tutor ha sido pieza clave para seleccionar en } \\
\text { forma adecuada tus asignaturas en cada ciclo } \\
\text { escolar. }\end{array}$ & $90(27)$ & $93(28)$ & $183(55)$ \\
\hline $\begin{array}{l}\text { Tu tutor te canaliza a las instancias adecuadas } \\
\text { cuando tienes algún problema que rebasa su área de } \\
\text { acción. }\end{array}$ & $80(24)$ & $109(33)$ & $189(57)$ \\
\hline $\begin{array}{l}\text { Tomas acuerdos con tu tutor al iniciar cada ciclo } \\
\text { escolar para planear acciones a realizar durante } \\
\text { dicho periodo. }\end{array}$ & $88(26)$ & $100(30)$ & $188(56)$ \\
\hline $\begin{array}{l}\text { Tu tutor te ha retroalimentado con aspectos que } \\
\text { tienes que fortalecer en tu formación académica. }\end{array}$ & $76(23)$ & $121(36)$ & $197(59)$ \\
\hline
\end{tabular}

Fuente: datos recopilados por los autores (2015).

En las categorías formación académica y valoración de las actividades realizadas por el tutor, $58 \%$ de los tutorados está de acuerdo con los conocimientos y actividades que realiza, lo cual representa — - según la escala establecida en esta investigación- un desempeño bueno del tutor en relación a sus funciones.

\section{CATEGORÍA AFECTIVA}

Se hicieron cinco preguntas acerca de la actitud del tutor, donde cuatro obtuvieron un porcentaje mayor al $60 \%$. El mayor, de $84 \%$ (suma de totalmente de acuerdo y de acuerdo) era sobre el trato hacia los tutorados con respeto y atención, seguido del $70 \%$ en la disposición para atenderlos y 69 \% en comunicación. Es importante resaltar que solo 33 $\%$ de los estudiantes manifestó que la primera persona a la que acudirían para comunicarle y pedirle apoyo en problemas familiares y emocionales sería a su tutor (tabla III). 
Tabla III: Indicador de la actitud del tutor de medicina

\begin{tabular}{lccccc}
\hline & $\begin{array}{c}\text { Acudo } \\
\text { primero a } \\
\text { mi tutor } \\
\text { cuando } \\
\text { tengo } \\
\text { problemas }\end{array}$ & $\begin{array}{c}\text { Muestra } \\
\text { interés y me } \\
\text { escucha }\end{array}$ & $\begin{array}{l}\text { Muestra } \\
\text { disposición }\end{array}$ & $\begin{array}{c}\text { Me trata con } \\
\text { respeto y } \\
\text { atención }\end{array}$ & $\begin{array}{c}\text { Es fácil } \\
\text { comunicarme } \\
\text { con él cuando } \\
\text { le expongo } \\
\text { mis } \\
\text { problemas }\end{array}$ \\
\hline $\begin{array}{c}\text { Totalmente } \\
\text { de acuerdo } \\
\mathbf{f}(\%)\end{array}$ & $48(14)$ & $91(27)$ & $98(29)$ & $170(51)$ & $112(33 \%)$ \\
\hline $\begin{array}{c}\text { De acuerdo } \\
\mathbf{f}(\%)\end{array}$ & $64(19)$ & $123(37)$ & $137(41)$ & $109(33)$ & $120(36 \%)$ \\
\hline $\begin{array}{l}\text { Total } \\
\mathbf{f}(\%)\end{array}$ & $112(33)$ & $214(64)$ & $235(70)$ & $279(84)$ & $232(69)$ \\
\hline
\end{tabular}

Fuente: datos recopilados por los autores (2015).

Al agrupar las cinco preguntas para explorar lo que perciben los estudiantes sobre la actitud del tutor, se encontró que 47 \% la percibe como regular según la valoración establecida.

A la pregunta de si los tutores son fácilmente localizables, $61 \%$ de los tutorados respondió que sí (suma de totalmente de acuerdo y de acuerdo), y $39 \%$ (suma de en desacuerdo y totalmente en desacuerdo) que no (gráfica 2). 


\section{Gráfica 2: ¿Localizas fácilmente a tu tutor?}

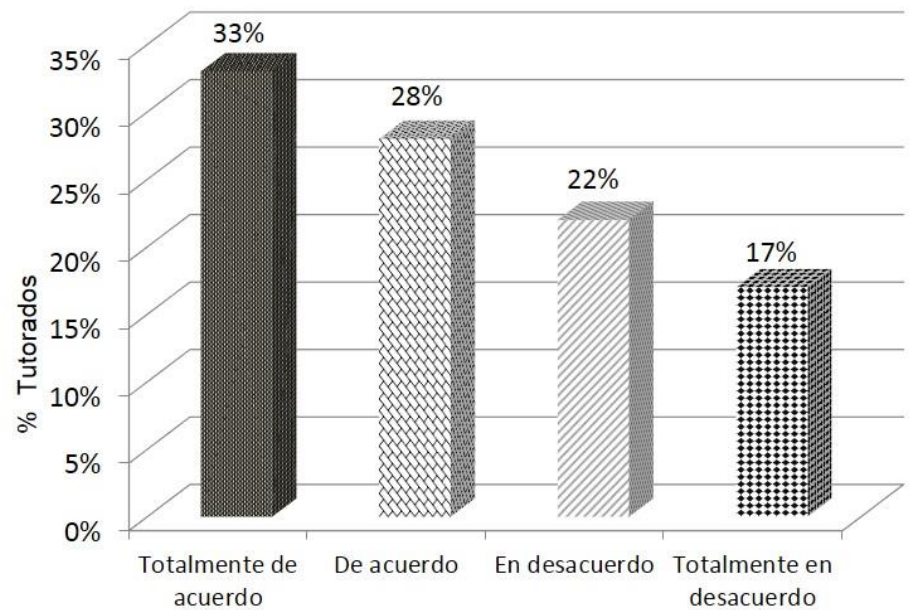

Fuente: datos recopilados por los autores (2015).

\section{DISCUSIÓN}

En la mayoría de las instituciones educativas de nivel superior se ha establecido la figura del tutor ya que a través de su desempeño (académico y afectivo) se pueden lograr los beneficios planteados por la ANUIES, por ejemplo, mejorar la calidad educativa y los indicadores de permanencia y eficiencia terminal de los estudiantes (ANUIES, 2000). Por tal motivo, es responsabilidad de la administración escolar capacitar, sensibilizar y evaluar el desempeño de los tutores periódicamente para la mejora continua del Programa Institucional de Tutorías. Los resultados de esta investigación muestran que 51 a $71 \%$ de los tutores tienen un buen desempeño respecto a su quehacer académico con los tutorados; sin embargo, en el aspecto afectivo, 26 a $50 \%$ manifestó que los tutores presentan una actitud regular para realizar su actividad tutorial.

Con respecto al primer aspecto, el estudio realizado por Orduño y Velazco (2009), mostró que $77.8 \%$ de los tutorados están de acuerdo con las orientaciones y asesorías por parte de los tutores, cifra superior a la encontrada en este estudio. 
En otro estudio realizado por Herrera y Guevara (2010) de la Facultad de Medicina de la UNAM, el aspecto afectivo (relación con su tutor de confianza y empatía) fue mejor calificado por $78 \%$ de los tutorados.

Marín y Sansores (2014), encontraron que la percepción de las tutorías por parte de los tutorados, en general, es que es útil en aspectos académicos, y aunque el aspecto afectivo con respecto al trato que reciben fue aceptable, obtuvo un puntaje más bajo, un resultado similar al encontrado en el presente trabajo.

Específicamente en un reporte realizado por Díaz, Marín, Salazar et al (2011), $97.6 \%$ de los tutorados consideran que el tutor es importante para su desempeño en actividades académicas; sin embargo, 70 \% manifestó que el tutor debe ser congruente con su actitud. En esta investigación, "la actitud del tutor" la perciben los tutorados como regular. Dicha percepción, aunada a las otras interpretaciones, deduce que los estudiantes acuden a los tutores principalmente para aspectos académicos administrativos, mientras que la relación afectiva con ellos, a pesar de ser aceptable, requiere de mayor habilidad para contribuir a la formación integral de los tutorados.

Knight y Lazaro (citados por Martínez, Pérez y Martínez, 2014), coinciden que el tutorado debe tener la suficiente confianza con su tutor para que este pueda ejercer mejor su actividad tutorial e influir positivamente en él. En este trabajo se encontró que solo $33 \%$ de los tutorados le tienen confianza a su tutor. Dichos autores señalan la importancia del afecto, la empatía y las relaciones interpersonales como competencias del tutor.

De acuerdo con Hernández, Rodríguez, Viñas y Ridal (2007), es necesaria una formación continua del tutor en los aspectos pedagógico y metodológico para que pueda guiar acertadamente a los estudiantes. Según estos autores, más del $60 \%$ de los tutores solo cuentan con formación disciplinar, por lo que no están preparados para realizar una función tutelar.

A pesar de la obligatoriedad que establece la universidad pública de que los tutores reciban previamente el curso de tutoría, es insuficiente la capacitación para que el programa logre su propósito. Son deseables el seguimiento permanente mediante la reflexión de los involucrados para la mejora de sus funciones y la motivación por parte de la institución educativa. Es necesario que se modifique la función del tutor para que se ajuste a las 
necesidades de los estudiantes y las tutorías se hagan con una actitud positiva, de confianza y respeto inherente a la práctica docente, de tal forma que se brinde asesoría integral en la formación del estudiante.

\section{CONCLUSIÓN}

De acuerdo a la percepción de los estudiantes, el desempeño del tutor de medicina en una escuela pública se considera bueno académicamente hablando (su conocimiento y capacidad de tutoría), pero regular en cuanto a la actitud que muestra al realizarla.

Entre las funciones del tutor más valoradas por los tutorados fue su labor de dirigir las trayectorias académicas con $64 \%$, así como su parte afectiva (actitud, o sea, atención y respeto) con $84 \%$. Por el contrario, la que menos valoraron fue el acudir con su tutor para plantearle problemas familiares y personales, lo cual habla de desconfianza hacia el tutor, con $33 \%$ (suma de totalmente de acuerdo y de acuerdo).

Se recomienda motivar, preparar y evaluar el proceso de tutorías, así como medir el impacto que este programa ha tenido en los indicadores de calidad educativa, para poder encauzar el programa institucional dentro del área médica. 


\section{Bibliografía}

ANUIES (2000). Programas Institucionales de Tutorías, una propuesta de la ANUIES para su organización y funcionamiento en las Instituciones de Educación Superior, México, ANUIES.

Chiavenato, I. (2000). Administración de recursos humanos. Santa Fe de Bogotá: Editorial McGraw-Hill.

Caballero, L.B. y Añorga, J. (2007). El modelo del tutor de especialidades médicas del ISMM “Dr. Luis Díaz Soto”. Revista de Educación Médica. Recuperado de: http://scielo.sld.cu/scielo.php?script=sci_arttext\&pid=S0864-21412007000200009

De la Cruz, G. Chehaybar, E. y Abreu L. (2011). Tutoría en Educación Superior: Una revisión analítica de la literatura. Revista de la Educación Superior. México. Facultad de Medicina UNAM. Recuperado en: http://www.scielo.org.mx/scielo.php?script=sci_arttext\&pid=S018527602011000100009

DACS (2012). Programa de tutoría de la Licenciatura de Médico Cirujano de la División Académica de Ciencias de la Salud de la Universidad Juárez Autónoma de Tabasco.

$\begin{array}{lll}\text { Villahermosa, } & \text { Tabasco. } & \text { Recuperado }\end{array}$ http://www.archivos.ujat.mx/2012/dacs/Lic_MC/PROGRAMAS,\%20MANUALES \%20Y\%20GUIAS/PROGRAMAS/PROGRAMA\%20TUTORIAS.pdf

Díaz Solís M. del R., Marín Chagoya M. A., Salazar Peña M.T. L., Martínez Reyes M. C., Montes de Oca Chávez N. (2011). Evaluación de la tutoría académica desde la perspectiva estudiantil. Facultad de Enfermería de la Benemérita Universidad Autónoma de Puebla. Investigación de la tutoría académica en el siglo XXI. Puebla. Disponible

en: http://sifcc.cs.buap.mx/SistemaIntegralTutorias/Productos/Libros/LibroT2011.pdf

Hernández, S. Rodríguez, M. Viñas, J. y Ridal, J. (2007). Papel del tutor en el internado profesionalizante. Revista de Ciencias Médicas de Pinar del Río. Recuperado en: http://scielo.sld.cu/scielo.php?script=sci_arttext\&pid=S1561-31942007000200004

Herrera Zamorano B. Guevara Guzmán R. (2010). La tutoría en la Facultad de Medicina. Conferencia del Encuentro Universitario tutorías. UNAM. México. Disponible en: 
http://www.tutoria.unam.mx/EUT2010/memoriaEUT/htmls/memoria/HTMLS/Eje4/ HerreraZamoranoBeatriz.pdf

Lobato, C. e Ilvento, M. (2013). La orientación y tutoría universitaria: una aproximación actual. Revista de Docencia Universitaria. Argentina: Universidad Nacional de Tucumán. Recuperado en: http://red-u.net/redu/index.php/REDU/article/view/646

Martínez C, Pérez J, Martínez M. (2014). Una revisión de la tutoría: la percepción de estudiantes y de tutores de estudio de grado. Vol. 12 (1), 269-305 p. Disponible en: http://red-u.net/redu/index.php/REDU/article/view/544

Marín L. y Sansores Valencia S. (2014). Evaluación de la tutoría en la División de Ciencias Políticas y Humanidades de la Universidad de Quintana Roo. Evaluación Debate.

Narro Robles, José, y Arredondo Galván, Martiniano. (2013). La tutoría: un proceso fundamental en la formación de los estudiantes universitarios. Perfiles educativos, 35(141), 132-151. Recuperado en 03 de abril de 2016, de http://www.scielo.org.mx/scielo.php?script=sci_arttext\&pid=S018526982013000300009\&lng=es\&tlng=es

Orduño Solís E., y Velazco Borques F. (2009). Evaluación de la efectividad del programa de tutorías en una Institución de Educación Superior en México 0705. Conferencia llevada a cabo en el X Congreso Nacional de Investigación Educativa. Consejo Mexicano de Investigación Educativa. Veracruz, México. Disponible en: http://www.comie.org.mx/congreso/memoriaelectronica/v10/pdf/area_tematica_13/ ponencias/0705-F.pdf

Real Academia Española (2016). Recuperado de http://dle.rae.es/?id=CqRHucK.

UJAT (2003). Programa institucional de tutorías de la Universidad Juárez Autónoma de Tabasco. Villahermosa, Tabasco. Recuperado de http://www.ujat.mx/Contenido/InteriorAdentro/270/2819

UJAT (2003). Evaluación del Programa Institucional de Tutorías. Anexo 4. Instrumento de Evaluación de los tutorados. Desempeño del tutor. Disponible en: http://www.archivos.ujat.mx/2011/dese/pit/3_instrumentos_evaluacion_tutoria.pdf

UNAM (2012). Programa Institucional de Tutoría. Facultad de Medicina. Cuestionario de Evaluación para los alumnos. Disponible en: http://www.facmed.unam.mx/sg/tutorias/docs/e_09.pdf 